\title{
UNITARY REPRESENTATIONS OF SOME LINEAR GROUPS
}

\author{
SEIZÔ ITÔ
}

§ 0. Introduction. Recently I. Gelfand and M. Neumark [2] have determined the types of irreducible unitary representations of the group $\mathbf{G}_{1}$ of linear transformations of the straight line. The analogous result is obtained for the group $\mathbf{G}_{2}$ of transformations $z \rightarrow a z+b$ in the complex-number plane $\mathbb{E}$, where $a$ and $b$ run over all complex numbers with the exception of $a=0$, which may be considered as the group of all sense-preserving similar transformations in the two-dimensional euclidean space $E^{2}$. In this paper, we shall determine the types of cyclic ${ }^{1)}$ unitary representations and irreducible unitary representations of the group $\mathbf{G}$ of all sense-preserving congruent transformations in $E^{2}$, which may be realized as the group of all transformations in 5 of the form $z \rightarrow a z+b ; a, b \in(5$ and $|a|=1$, The method is due to the same idea as GelfandNeumark's one [2], but we need Lemma $2(\S 2)$ which is not necessary in the case of $\mathbf{G}_{1}$ and of $\mathbf{G}_{2}$. Our method may be applied to the group $\mathbf{G}^{\prime}$ of all transformations $q \rightarrow a q+b$ in the field $\mathbf{Q}$ of quaternions, where $a, b \in \mathbf{Q}$ and $\|a\|=1 .^{2)}$

The author expresses his hearty thanks to Prof. K. Yosida, Mr. H. Yoshizawa and Mr. S. Murakami who have encouraged him with kind discussions.

$\S 1$. Main results. Let $\mathbf{G}$ be the group of all transformations $z \rightarrow a z+b$ in the

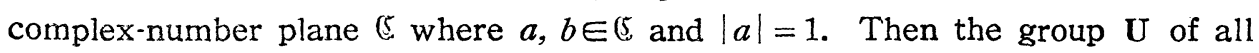
rotations $z \rightarrow a z,|a|=1$, is a subgroup of $\mathbf{G}$ and the group $\mathbf{V}$ of all translations $z \rightarrow z+b$ is a commutative normal subgroup of $\mathbf{G}$, and it holds that

$$
\left\{\begin{array}{l}
\mathbf{G}=\mathbf{U} \cdot \mathbf{V}, \mathbf{U} \cap \mathbf{V}=\{e\} \quad(e=\text { the identity of } \mathbf{G}), \\
\mathbf{G} / \mathbf{V} \cong \mathbf{U} .
\end{array}\right.
$$

Hereafter we shall denote by $u_{a}$ and $v_{b}$ the elements of $\mathbf{U}$ and $\mathbf{V}$ corresponding to the complex number $a(|a|=1)$ and $b$ respectively. Then we have $u_{1}=v_{0}$ $=e$ and

Received September 17, 1951.

1) It is called "simple" in [3].

2) The group $G^{\prime}$ is different from the group of all sense-preserving congruent transformations in $E^{4}$. It seems to be more complicated to determine the types of unitary representations of the group of all sense-preserving congruent transformations in $E^{n}$ for $n \geqq 3$; - see $\S 4$. 
$u_{a} v_{b}=v_{a b} u_{a}$.

Let $X$ be the character group of $\mathbf{v}$ and $\chi_{0}$ be the identity character. Then $X$ is isomorphic to the two-dimensional vector group as well as $\mathbf{V}$ and consequently every element $\chi$ of $X$ may be considered as a complex number rexp $(i \theta)(r \geq 0)$. Hereafter we shall denote every $\chi \equiv r \exp (i \theta) \in X$ by the couple $\langle s, r\rangle$ where $s=\exp (i \theta)$; such a couple is unique for $\chi \neq \chi_{0} \equiv 0$, and $\widetilde{X}=X-\left\{\chi_{0}\right\}$ is the topological product space of the unit circle $S$ in the complex-number plane and $R=(0, \infty)$. Thus we may consider the transformations $\chi \rightarrow a \chi$ in $X$ and $s \rightarrow a s$ $(|a|=1)$ in $S$ as the multiplication of complex numbers.

We shall here state the main theorems.

THEOREM 1. Let $\sigma(\Gamma)(I \subset S)$ be the measure on $S$ invariant under rotations:-

i) Fix an arbitrary element $\gamma_{0} \in R$, and define the unitary operator $U(g)$ $(g \in \mathbf{G})$ in the Hilbert space $\mathscr{H}=L^{2}(S, \sigma)$ as follows: $U_{a} \psi(s)=\psi\left(a^{-1} s\right), V_{b} \psi(s)$ $=\left(b,\left\langle s, r_{0}\right\rangle\right) \psi(s)^{3)}\left(\psi(s) \in L^{2}(S, \sigma)\right)$ and $U(g)=U_{a} V_{b}$ for $\left.g=u_{a} v_{b}{ }^{4}\right)$ Then $\{\xi$, $U(g)\}$ is an irreducible unitary representation of $\mathbf{G}$, and for any fixed $\psi_{0}(s)$ $\in L^{2}(S, \sigma)$ such that $\left\|\psi_{0}\right\|=1$ the function

$$
\Phi(g) \equiv \emptyset\left(u_{a} v_{b}\right)=\int_{s}\left(b,\left\langle a^{-1} s, r_{0}\right\rangle\right) \psi_{0}\left(a^{-1} s\right) \overline{\phi_{0}(s)} d \sigma(s) \quad\left(g=u_{a} v_{b}\right)
$$

is the normal elementary ${ }^{6)}$ p. $d .^{6)}$ function on $\mathbf{G}$ corresponding to the above irreducible unitary representation.

ii) If $r_{1}, r_{2} \in R$ and $r_{1} \neq r_{2}$, then the unitary representation as stated in i) corresponding to $r_{1}$ is not unitary equivalent to that corresponding to $r_{2}$.

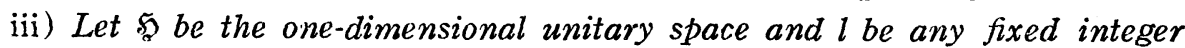
( $\equiv 0)$, and define the unitary operator $U(g)$ by $U_{a} \psi=a^{l} \psi, V_{b} \psi=\psi(\psi \in \mathfrak{S})$ and $U(g)=U_{a} V_{b}$ for $g=u_{a} v_{b}$. Then $\{\mathfrak{g}, U(g)\}$ is an irreducible unitary representation of $\mathbf{G}$, and

$$
\Phi(g) \equiv \Phi\left(u_{a} v_{b}\right)=a^{l} \equiv \exp (i l \theta) \quad(\text { for } a=\exp (i \theta))
$$

is the corresponding normal elementary p. d. function on $\mathbf{G}$.

iv) Every irreducible unitary representation of $\mathbf{G}$ is unitary equivalent to one of the above stated types. Consequently every normal elementary $p . d$. function on $\mathbf{G}$ is expressible in the form (1.3) or (1.4).

ThEOREM 2. Let $\sigma(T)$ be as stated in Theorem 1 , and $\rho_{j}(\Delta)(\Delta \subset R), j=1$,

3) $(b, \chi)$ denotes the value of character $\chi(\in X)$ at the element $v_{b} \in \mathbf{V}$.

4) Any element $g \in G$ is uniquely expressible in this form by virtue of (1.1) and (1.2).

5) See [3] $\$ 15$.

B) Abbreviated for positive definite. 
$2, \ldots, n(\leqq \infty)$, be measures on $R$ such that $\rho_{j}(R)<\infty ;-$

i) In every Hilbert space $\mathfrak{N}_{j}=L^{2}\left(\widetilde{X}, \sigma \otimes \rho_{j}\right)$, ${ }^{7}$ we define the unitary operator $U(g) \quad(g \in \mathbf{G})$ as follows: $U_{a} \psi(s, r)=\psi\left(a^{-1} s, r\right) \quad V_{b} \psi(s, r)=(b,\langle s, r\rangle) \psi(s, r)$ $\left(\phi(s, r) \in L^{2}\left(\tilde{X}, \sigma \otimes \rho_{j}\right)\right)$ and $U(g)=U_{a} V_{b}$ for $g=u_{a} v_{b}$; and let $f_{j}(s, r), j=1$, $2, \ldots, n(\leqq \infty)$, be functions as follows :

$\left.1^{\circ}\right) f_{j}(s, r) \in L^{2}\left(\tilde{X}, \sigma \otimes \rho_{j}\right)$ for every $j$,

$\left.2^{\circ}\right) \int_{S}\left|f_{j}(s, r)\right|^{2} d \sigma(s)=1$ for $\rho_{j}$-almost all $r$,

$\left.3^{\circ}\right) f_{j}(s, r) / f_{k}(s, r)$ is not constant essentially $(\sigma)$ as a function of $s$ for $\rho_{j}$ - or $\rho_{k^{-}}$almost all $r$.

Let $\left\{\Re_{l}, U_{l}(g)\right\}$ be the irreducible unitary representation of $\mathbf{G}$ as stated in Theorem 1 ii) corresponding to the integer $l, f_{l}^{\prime}$ be an arbitrarily fixed element of $\mathfrak{N}_{l}$, and $\left\{l_{1}, l_{2}, \ldots, l_{N}\right\}(N \leqq \infty)$ be a sequence of integers such that $k \neq j$ implies $l_{k} \neq l_{j}$. Then any of $\left\{\mathfrak{M}_{j}, U(g), f_{j}\right\}(j=1,2, \ldots, n)$ and $\left\{\mathfrak{g}_{\delta}, U(g)\right.$, $\left.f^{\circ}\right\}$ defined by

and

$$
\{\mathfrak{g}, U(g)\}=\left[\bigoplus_{j=1}^{n}\left\{\mathfrak{M}_{j}, U(g)\right\}\right] \oplus\left[\bigoplus_{k=1}^{N}\left\{\Re_{l_{i}}, U_{l_{k}}(g)\right\}\right]^{8)}
$$

$$
f^{\circ}=\sum_{j=1}^{n} \alpha_{j} f_{j}+\sum_{k=1}^{N} \beta_{k} f_{l_{k}}^{\prime 9)} \quad\left\{\begin{array}{l}
\sum_{j=1}^{n}\left|\alpha_{j}\right|^{2}<\infty(\text { if } n=\infty) \\
\sum_{k=1}^{N}\left|\beta_{k}\right|^{2}<\infty(\text { if } N=\infty)
\end{array}\right.
$$

are cyclic unitary representations of $\mathbf{G}$. The $p$. $d$. function $\Psi(g)$ corresponding to the unitary representation $\left\{\mathfrak{H}, U(g), f^{\circ}\right\}$ is as follows:

$$
\begin{aligned}
& \Psi(g) \equiv \Psi\left(u_{a} v_{b}\right) \\
& =\sum_{j=1}^{n} A_{j} \int_{R} d \rho_{j}(r) \int_{S}\left(b,\left\langle a^{-1} s, r\right\rangle\right) f_{j}\left(a^{-1} s, r\right) \overline{f_{j}(s, r)} d \sigma(s) \\
& +\sum_{k=1}^{\infty} B_{k} \exp \left(i l_{k} \theta\right) \\
& \text { for } g=u_{a} v_{b}, a=e^{i \theta} . \\
& \left(A_{j}=\left|\alpha_{j}\right|^{2}, \quad B_{k}=\left|\beta_{k}\right|^{2}\right) \text {. }
\end{aligned}
$$

ii) Every cyclic unitary representation of $\mathrm{G}$ is unitary equivalent to that of above stated type, and any p. d. function on $\mathbf{G}$ is expressible in the form (1.5), where $0 \leqq n \leqq \infty$ and $0 \leqq N \leqq \infty$. The functions

$$
\begin{aligned}
\Phi_{j}(g ; r) & \equiv \Phi_{j}\left(u_{a} v_{b}, r\right) \\
& =\int_{s}\left(b,\left\langle a^{-1} s, r\right\rangle\right) f_{j}\left(a^{-1} s, r\right) \overline{f_{j}(s, r)} d_{\sigma}(s) \\
& (r \in R ; j=1,2, \ldots)
\end{aligned}
$$

7) $\sigma \otimes \rho_{j}$ denotes the product measure of $\sigma$ and $\rho_{j}$.

8) See [3] $\$ 5$ as for the direct sum of unitary representations.

9) The right-hand side means the summation as elements of the Hilbert space $\mathfrak{5}$. 
and

$$
\begin{aligned}
\chi_{l}(g) \equiv \chi_{l}\left(u_{a} v_{b}\right)= & \exp (i l \theta) \text { for } a=e^{i \theta} \\
& (l=\ldots,-2,-1,0,1,2, \ldots)
\end{aligned}
$$

are normal elementary $p$. d. functions on $\mathbf{G}$ and any $p$. d. function $\Psi(g)$ is expressible in the form

$$
\Psi(g)=\sum_{j=1}^{\infty} A_{j} \int_{R} \emptyset_{j}(g ; r) d \rho_{j}(r)+\sum_{l=-\infty}^{\infty} B_{l} \chi_{l}(g),
$$

where $A, B \gg 0, \sum_{j=1}^{\infty} A_{j} \rho_{j}(R)<\infty$ and $\sum_{l=-\infty}^{\infty} B_{l}<\infty$. (Cf. Bochner-Raikov's theorem for p. d. functions on commutative groups.)

As for the group $\mathbf{G}^{\prime}$ of all transformations $q \rightarrow a q+b,\|a\|=1$, in the field $\mathbf{Q}$ of quaternions, any irreducible unitary representation and any cyclic unitary representation of $\mathbf{G}^{\prime}$ may be obtained by the same methods as stated in Theorems 1 and 2, where the irreducible unitary representation stated in Theorem 1 iii) must be replaced by an irreducible unitary representation of the compact group of all transformations $q \rightarrow a q(\|a\|=1)$ in $Q$; such modifications are necessary for cyclic unitary representations.

After some preliminaries in $\S 2$, we shall prove Theorem 1 in $\S 3$ and Theorem 2 in $\S 4$. Some supplementary remarks will be also given in $\S 4$.

\section{§2. Preliminary lemmas.}

LEMMA 1. Let $\{\mathfrak{M}, U(x)\}$ be a unitary representation (not necessarily cyclic) of the n-dimensional vector group $\mathbf{x}$, where $\mathfrak{M}$ is a separable Hilbert space. Then there exists a resolution of the identity $\{E(\Lambda)\}$ in $\mathfrak{M}$ on the character group $X$ of the group $\mathbf{X}$ such that

$$
U(x)=\int_{X}(x, \%) d E(\%) .
$$

Further the space $\mathfrak{M}$ can be realized as an at most countable direct sum of spaces $\mathfrak{M}_{j}(j=1,2, \ldots)$ of the function $f_{j}(\%)$ such that

$$
\left\|f_{j}\right\|=\int_{X}\left|f_{j}(\chi)\right|^{2} d F_{j}(\%)<\infty
$$

where $F_{j}(A)$ is a measure on $X$ such that $F_{j}(X)=1$ and every $F_{j}(A)$ is absolutely continuous with respect to $F_{j-1}(\Lambda)(j>1)$; furthermore, if $f \in \mathfrak{M}$ is realized by $\left\{f_{j}(\chi) / j=1,2, \ldots\right\}$, then $U(x) f$ by $\left\{(x, \chi) f_{j}(\chi) / j=1,2, \ldots\right\}$.

This lemma is well known as Stone's theorem and Hahn-Hellinger's theory ${ }^{10}$ in the case $n=1$, and may be proved in our general case by the same idea.

Lemma 2, Let $\tilde{X}, R$ and $S$ be as stated in $\S 1$ and $F(A)(A \subset \widetilde{X} \equiv S \times R)$ be a measure on $\tilde{X}$ such that $F(\widetilde{X})<\infty$, and assume that there exists a non-nega-

10) See [5] Chapter VII. 
tive function $u(a ; \chi)$ on $S \times \tilde{X}(a \in S, \chi \in \tilde{X}), B$-measurable in $\langle a, \chi\rangle$ and summable on $\widetilde{X}$ with respect to the measure $F(\Lambda)$ for every $a \in S$, such that

$$
F\left(a^{-1} \Lambda\right)=\int_{\Lambda} u(a ; \chi) d F(\chi)^{11)}
$$

for any $A \subset \tilde{X}$ and any $a \in S$. Then there exist a non-negative $B$-measurable function $\omega(s, r)$ on $\widetilde{X}=S \times R$ and a measure $\rho(\Delta)$ on $R, \rho(R)<\infty$, such that $F(\Lambda)$ is given by

$$
F(\Lambda)=\int_{\Lambda} \omega(s, r) d \sigma(s) d \rho(r)
$$

where $\sigma(\Gamma)$ is the measure on $S$ invariant under rotations.

Proof. For any fixed $\Delta \subset R, F_{\Delta}(\Gamma)=F(\Gamma \times \Delta)(\Gamma \subset S)$ is a measure on $S$ and it follows from the assumption (2.1) that $F_{\Delta}(a \Gamma)$ is absolutely continuous with respect to $F_{\Delta}(\Gamma)$ for every $a \in S$. Hence $F_{\Delta}(\Gamma)$ is absolutely continuous with respect to the invariant measure $\sigma(\Gamma){ }^{12)}$ And hence there exists a function $\mu(s, \Delta)$ of a point $s \in S$ and a set $\Delta C R$ such that

i) for any fixed $s \in S, \mu(s, \Delta)$ is a regular measure on $R$ and $\mu(s, R)<\infty$,

ii) for any fixed $\Delta \subset R, \mu(s, \Delta)$ is B-measurable in $s$, and

iii) for any $I \subset S$ and $\Delta \subset R, F(\Gamma \times \Delta)=\int_{\Gamma} \mu(s, \Delta) d \sigma(s)$; this fact is proved Dy J. L. Doob [1] as the existence- and uniqueness-theorem of the conditional probability law. Consequently for any $\varphi(\chi) \equiv \varphi(s, r) \in L^{1}(\tilde{X}, F)$, we have

$$
\int_{\widetilde{X}} \varphi(s, r) d F(\chi)=\int_{S} d \sigma(s) \int_{R} \varphi(s, r) \mu(s, d r) ;
$$

the iterated integral in the right-hand side is well defined by i) and ii), and this equals the left-hand side by iii). From (2.1) and (2.3), we get

$$
\begin{aligned}
\int_{\Gamma} \mu(a s, \Delta) d \sigma(s) & =F\left(a^{-1} \Gamma \times \Delta\right)=\int_{\Gamma \times \Delta} u(a ; \chi) d F(\chi) \\
& =\int_{\Gamma} d \sigma(s) \int_{\Delta} u(a ; s, r)_{\mu}(s, d r)
\end{aligned}
$$

for any $T \subset S, \Delta \subset R$ and any $a \in S$, where $u(a ; s, r)=u(a ; \chi)$ for $\chi=\langle s, r\rangle$. And hence, for any $\Delta$, we have

$$
\mu(a s, \Delta)=\int_{\Delta} u(a ; s, r) \mu(s, d r) \quad \text { for } \sigma \text {-almost all } s \in S .
$$

By Fubini's theorem, (2.4) is true for $\sigma$-almost all $a$ for $\sigma$-almost all s. Since the space $R$ has countable open bases and since $\mu(s, \Delta)$ is a regular measure

11) $a^{-1} \Lambda=\left\{a^{-1} \chi / \chi \in \Lambda\right\} ;-$ see $\S 1$.

12) This fact is well known as $D$. Raikov's lemma. 
on $R$ for every $s$, there exists a point $s_{0} \in S$, independent of $\Delta$, such that

$$
\mu\left(a s_{0}, \Delta\right)=\int_{\Delta} u\left(a ; s_{0}, r\right) \mu\left(s_{0}, d r\right) \quad \text { for } \sigma \text {-almost all } a \in S .
$$

Since the transformation $a \rightarrow a s_{0}^{-1}$ is measure-preserving, we obtain by putting $a=s s_{0}^{-1}$ that

$$
\mu(s, \Delta)=\int_{\Delta} u\left(s s_{0}^{-1} ; s_{0}, r\right) \mu\left(s_{0}, d r\right) \quad \text { for } \sigma \text {-almost all } s \in S .
$$

If we put $\omega(s, r)=u\left(s s_{0}^{-1} ; s_{0}, r\right)$ and $\rho(\Delta)=\mu\left(s_{0}, \Delta\right)$, then $\omega(s, r)$ is B-measurable in $\langle s, r\rangle$ and, by (2.3), (2.4) and Fubini's theorem, we have

$$
\begin{aligned}
\int_{\widetilde{x}} \varphi(s, r) d F(\chi) & =\int_{s} d \sigma(s) \int_{R} \varphi(s, r) \omega(s, r) d \rho(r) \\
& =\int_{\widetilde{X}} \varphi(s, r) \omega(s, r) d \sigma(s) d \rho(r)
\end{aligned}
$$

for any $\varphi \in L^{1}(\widetilde{X}, F)$; this implies (2.2), q.e.d.

LEMMA 3. Let $\mathrm{U}, \mathrm{V}$ and $\tilde{X}$ etc. be as in Theorem $2, f_{1}(s, r)$ be a function $\in L^{2} \equiv L^{2}\left(\tilde{X}, \sigma \otimes \rho_{1}\right)$ such that $\sigma\left(\left\{s / f_{1}(s, r) \neq 0\right\}\right)>0$ for $\rho_{1}$-almost all $r \in R$, and $L$ be the totality of linear combinations of the functions of the form $(b,<s$, $r\rangle) f_{1}\left(a^{-1} s, r\right),|a|=1$. Then $L$ is dense in $L^{2}$ with respect to the norm in $L^{2}$.

Proof (outline). For any set $A \subset \widetilde{X}$ and any $r \in R, A_{r}$ denotes the set $\{s \mid\langle s, r\rangle \in A\}$ by definition. Let $\Delta$ be any fixed subset of $R$. If $\sigma\left(A_{r}\right)>0$ for $\rho_{1}$-almost all $r \in \Delta$ and $A^{\prime} \subset S \times \Delta$, then there exist $u_{a_{1}}, \ldots, u_{a_{n}} \in \mathbf{U}$ for any $\varepsilon>0$ such that $\sigma \otimes \rho_{1}\left(\Lambda^{\prime}-\left[a_{1} \Lambda \cup \ldots \cup a_{n} \Lambda\right]\right)<\varepsilon$. On the other hand, any continuous function on $\widetilde{X}$ is approximated uniformly on any compact subset of $\tilde{X}$ by means of linear combinations of characters. By making use of these facts, we may prove that any continuous function on $\tilde{X}$ which vanishes outside of a compact set is approximated in $L^{2}$ by means of functions $\in L$. Lemma 3 follows from this result at once.

§3. Proof of Theorem 1. Let $\mathbf{G}, \mathbf{U}$ and $\mathbf{V}$ etc. be as stated in Theorem 1 and $\left\{\mathfrak{g}, U(g), f^{\circ}\right\}$ be a cyclic unitary representation of $\mathbf{G}$, and put $U_{a}=U\left(\boldsymbol{u}_{a}\right)$ for $u_{a} \in \mathbf{U}$ and $V_{b}=U\left(v_{b}\right)$ for $v_{b} \in \mathbf{V}$. Then it follows from (1.2) that

$$
U_{a} V_{b}=V_{a b} U_{a} \text {. }
$$

Since $\mathbf{G}$ satisfies the second countability axiom and since the representation is cyclic, the Hilbert space $\mathscr{S}$ is separable. Put

$$
\mathfrak{R}=\left\{f \in \mathscr{S} / V_{b} f=f \text { for all } v_{b} \in \mathrm{V}\right\} .
$$

Then, since $\mathbf{V}$ is a normal subgroup of $\mathbf{G}, f \in \mathfrak{N}$ implies that $V_{b} U(g) f$ $=U(g) U\left(g^{-1} v_{b} g\right) f=U(g) f$ for any $g \in \mathbf{G}$ and $v_{b} \in \mathbf{V}$. Therefore $\mathfrak{R}$ and con- 
sequently $\mathfrak{M}=\mathfrak{S} \ominus \Re$ are $U(g)$-invariant subspaces of $\mathfrak{g}$. The representation, considered in $\Re$, yields a representation of the group $\mathbf{U}(\cong \mathbf{G} / \mathbf{V})$.

Consider the representation in $\mathfrak{M} ; \mathfrak{M}$ is separable as well as $\mathfrak{g}$. By Lemma 1 , there exists a resolution of the identity $\{E(\Lambda)\}$ in $\mathfrak{M}$ on $X$ such that

$$
V_{b}=\int_{X}(b, \chi) d E(\chi) ;
$$

and the space $\mathfrak{M}$ may be realized as an at most countable direct sum of the spaces $\mathfrak{M}_{j}$ of functions:

$$
\mathfrak{M}_{j}=\left\{f_{j}(\not) /\left\|f_{j}\right\|^{2}=\int_{X}\left|f_{j}(\chi)\right|^{2} d F_{j}(\chi)<\infty\right\},
$$

where $F_{j}(A)$ is a measure on $X$ such that $F_{j}(X)=1$ and every $F_{j}(A)(j>1)$ is absolutely continuous with respect to $F_{j-1}(A)$. When $f \in \mathfrak{M}$ is realized by $\left\{f_{j}(\chi)\right\}$, we write $f \sim\left\{f_{j}(\chi)\right\}$; then

$$
V_{b} f \sim\left\{(b, \chi) f_{j}(\chi)\right\}
$$

for any $v_{b} \in \mathbf{V}$.

Since 0 is the only one element of $\mathfrak{M}$ that fulfills $V_{b} f=f$ for all $v_{b} \in \mathbf{V}$ we obtain $F_{j}\left(\left\{\chi_{0}\right\}\right)=0, j=1,2, \ldots$ Thus we may consider $F_{j}(\Lambda), j=1,2, \ldots$, as measures on $\widetilde{X}=X-\left\{\chi_{0}\right\}$.

The operator $U_{a}$ is expressible as a matrix $\left(U_{j k}(a)\right)$ where $U_{j k}(a)$ is a bounded operator from $\mathfrak{M}_{k}$ into $\mathfrak{M}_{j}$ such that

$$
U_{a} \sim\left\{\sum_{k} U_{j k}(a) f_{k}(\chi)\right\}_{j=1,2}, \ldots \quad \text { for } f \sim\left\{f_{j}(\mathcal{X})\right\} .
$$

Since $U_{a}$ is unitary, we have

$$
\sum_{j}\left\|f_{j}\right\|^{i}=\sum_{j}\left\|\sum_{k} U_{j k}(a) f_{k}\right\|^{2} .
$$

Next, if we put $U_{j k}(a) \cdot 1=u_{j k}^{\circ}(a ; \%)$, then

$$
\left\|u_{j k}^{\circ}(a ; \chi)-u_{j k}^{\circ}(b ; \chi)\right\|^{2} \leqq\left\|U_{a} f^{i}-U_{b} f^{k}\right\|_{\xi}^{2} \quad(|a|=|b|=1),
$$

where $f^{k} \sim\left\{f_{j}(\chi)\right\}$ such that $f_{k}(\chi) \equiv 1$ and $f_{j}(\mathcal{H}) \equiv 0 \quad(j \neq k)$, and $\|\cdot\|_{\mathfrak{S}}$ denotes the norm in $\mathfrak{g}$; moreover $\mathbf{U}$ satisfies the second axiom of countability. Hence we may construct a function $u_{j k}(a ; \chi)$ B-measurable in $\langle a, \chi\rangle$ and such that $u_{j k}(a ; \chi)=u_{j k}^{\circ}(a ; \chi)$ for $F_{j}$-almost all $\chi$ for every $a{ }^{13)}$ Thus we may consider that $U_{j k}(a) \cdot 1=u_{j k}(a ; \chi)$. Then we get

$$
U_{j k}(a) f_{k}(\chi)=u_{j k}(a ; \chi) f_{k}\left(a^{-1} \%\right) .
$$

At first we can prove this equality for functions of the form $f_{k}(\chi)=(b, \chi)$ (for any fixed $b)$ by making use of (3.1), (3.2) and the fact that $(a b, \chi)=\left(b, a^{-1} \chi\right)$

13) Such $\boldsymbol{u}_{j k}(\boldsymbol{a} ; \chi)$ may be obtained by the same way as constructing the "measurable kernel" of a stochastic process. See [4]. 
$(|a|=1)$. Since the totality of linear combinations of "characters" $(b, \chi)$ is dense in $L^{2}\left(\widetilde{X}, F_{k}\right),(3.4)$ is true for all $f_{k} \in L^{2}\left(\widetilde{X}, F_{k}\right)$. Hence (3.3) becomes as follows:

$$
\sum_{j} \int_{\widetilde{X}}\left|f_{j}(\chi)\right|^{2} d F_{j}(\chi)=\sum_{j} \int_{\widetilde{X}}\left|\sum_{k} u_{j k}(a ; \chi) f_{k}\left(a^{-1} \chi\right)\right|^{2} d F_{j}(\chi) .
$$

Let $\varphi(\chi)$ be the characteristic function of $\Lambda \subset \widetilde{X}=S \times R$ and put in (3.5) $f_{1}(\chi)=\varphi(a \chi)$ and $f_{j}(\chi) \equiv 0$ for $j \neq 1$. Then we obtain

$$
\begin{aligned}
F_{1}\left(a^{-1} A\right) & =\int_{\widetilde{x}} \varphi(a \chi) d F_{1}(\chi)=\sum_{j} \int_{\widetilde{x}}\left|u_{j 1}(a ; \chi) \varphi(\chi)\right|^{2} d F_{j}(\chi) \\
& =\sum_{j} \int_{\Lambda}\left|u_{j 1}(a ; \chi)\right|^{2} d F_{j}(\chi) .
\end{aligned}
$$

Since all $F_{j}(\Lambda)$ are absolutely continuous with respect to $F_{1}(\Lambda)$ (by Lemma 1), we may write

$$
F_{j}(\Lambda)=\int_{\Lambda} \Phi_{j}(\chi) d F_{1}(\chi)
$$

where every $\Phi_{j}(\chi)$ is non-negative, B-measurable in $\chi$ and summable on $\tilde{X}$ with respect to $F_{1}$. Then the function

$$
u(a ; \chi)=\sum j\left|u_{j 1}(a ; \chi)\right|^{2} \Phi_{j}(\chi) \quad(\geqq 0)
$$

is B-measurable in $\langle a ; \chi\rangle$ and summable on $\tilde{X}$ with respect to $F_{1}$ for any $a$, and it follows from (3.6) and by Lebesgue's convergence theorem that

$$
F_{1}\left(a^{-1} \Lambda\right)=\int_{\Delta} u(a ; \chi) d F_{1}(\chi)
$$

Hence, by Lemma 2, there exist a non-negative B-measurable function $\omega(s, r)$ on $\widetilde{X}$ and a measure $\rho(\Delta)$ on $R$ such that $\rho(R)=1$ and $F_{1}(\Lambda)$ is given by

$$
F_{1}(\Lambda)=\int_{\Lambda} \omega(s, r) d \tau(s) d \rho(r)
$$

and consequently there exist non-negative B-measurable functions $\omega_{j}(s, r)$, $j=1,2, \ldots$, on $\tilde{X}=S \times R$ such that

$$
F_{j}(\Lambda)=\int_{\Lambda} \omega_{j}(s, r) d \sigma(s) d \rho(r)
$$

Now put $\Lambda_{j}=\left\{\langle s, r\rangle / \omega_{j}(s, r)=0\right\}$. Evidently $\Lambda_{1} \subset \Lambda_{2} \subset \ldots$ Put $\varphi_{j}(s, r)$ $=\omega_{j}(s, r) f_{j}(s, r)$ for every $f \sim\left\{f_{j}(s, r)\right\}$ and define the norm of $\varphi_{j}$ by

$$
\left\|\varphi_{j}\right\|^{2}=\int_{\widetilde{x}}\left|\varphi_{j}(s, r)\right|^{2} d \sigma(s) d \rho(r) .
$$

Then we have $\left\|\varphi_{j}\right\|^{2}=\left\|f_{j}\right\|^{2}$, and hence the mapping $f_{j} \rightarrow \varphi_{j}$ is an isometric mapping from $\mathfrak{M}_{j}$ onto 


$$
\mathfrak{R}_{j}=\left\{\varphi_{j}(s, r) /\left\|\varphi_{j}\right\|^{2}<\infty, \varphi_{j}(s, r)=0 \text { on } A_{j}\right\} .
$$

So we can realize $\mathfrak{M}$ as a direct sum of $\Omega_{j}$. The mapping $f_{j} \rightarrow \varphi_{j}$ carries $U_{j k}(a)$ into operators on $\left\{\varphi_{j}(s, r)\right\}$; we denote them by $U_{j k}(a)$ again. Define

$$
u_{j k}^{\prime}(a ; s, r)= \begin{cases}\omega_{j}(s, r) u_{j k}(a ; s, r) \omega_{k}\left(a^{-1} s, r\right)^{-1} & \text { if }\left\langle a^{-1} s, r\right\rangle \notin \Lambda_{k}, \\ 0 & \text { if }\left\langle a^{-1} s, r\right\rangle \in A_{k}\end{cases}
$$

$\left(u_{j k}(a ; s, r) \equiv u_{j k}(a ; \chi)\right.$ for $\left.\chi=\langle s, r\rangle\right)$. Then it follows from (3.4) and by the definition of $\varphi_{j}(s, r)$ that

$$
U_{j k}(a) \varphi_{k}(s, r)=u_{j k}^{\prime}(a ; s, r) \varphi_{k}\left(a^{-1} s, r\right),
$$

and unitary condition (3.5) becomes

$$
\begin{aligned}
\sum_{j} \int_{\widetilde{X}}\left|\varphi_{j}(s, r)\right|^{2} d \sigma(s) d \rho(r) & =\sum_{j} \int_{\widetilde{x}}\left|\sum_{k} u_{j k}^{\prime}(a ; s, r) \varphi_{k}\left(a^{-1} s, r\right)\right|^{2} d \sigma(s) d \rho(r) \\
& =\sum_{j} \int_{\widetilde{X}}\left|\sum_{k} u_{j k}^{\prime}(a ; a s, r) \varphi_{k}(s, r)\right|^{2} d \sigma(s) d \rho(r) .
\end{aligned}
$$

Denote by $\boldsymbol{n}(\leqq \infty)$ the number of $\mathfrak{M}_{j}$ and by $\mathfrak{S}_{0}$ the unitary space of all sequences $\xi=\left\{\xi_{j}\right\} \equiv\left\{\xi_{1}, \ldots, \xi_{n}\right\}$ of complex numbers such that $\|\xi\|^{2}=\sum_{j=1}^{n}\left|\xi_{j}\right|^{2}$ $<\infty$ (if $n=\infty)$ and by $\mathscr{S}_{k}(k=1,2, \ldots)$ the finite-dimensional subspace of $\mathscr{S}_{0}$ defined by the condition $\xi_{k}=\xi_{k+1}=\ldots=0 . \quad f \sim \varphi(\mathcal{X})=\left\{\varphi_{j}(s, r)\right\}$ means that $f \in \mathbb{M}$ is realized as a vector function $\varphi(\chi)$ such that $\varphi(\chi) \in \mathscr{S}_{0}$ for $\chi \notin \bigcup_{k=1}^{n} \Lambda_{k}$ and $\varphi(\chi) \in S_{\delta}$ for $\chi \in \Lambda_{k}$. Denote the matrix $\left(u_{j k}^{\prime}(a ; s, r)\right)$ by $M(a ; s, r)$ for every $\langle a ; s, r\rangle$. Then $f \sim \varphi(\mathcal{K}) \equiv \varphi(s, r)$ implies that

$$
\left\{\begin{array}{l}
\|f\|_{\tilde{Q}}^{2}=\int_{\tilde{x}}\|\varphi(s, r)\|^{2} d \sigma(s) d \rho(r) \quad\left(\|\varphi(s, r)\|^{2}=\sum_{j}\left|\varphi_{j}(s, r)\right|^{2}\right) \\
U_{a f} \sim M(a ; s, r) \varphi\left(a^{-1} s, r\right) \\
V_{b} f \sim(b,\langle s, r\rangle) \varphi(s, r)
\end{array}\right.
$$

by (3.2), (3.9) and the definition of $\varphi_{j}(s, r)$.

(3.10) is now written as follows:

$$
\int_{\widetilde{X}}\|\varphi(s, r)\|^{2} d \sigma(s) d \rho(r)=\int_{\widetilde{X}}\|M(a ; a s, r) \varphi(s, r)\|^{2} d \sigma(s) d \rho(r) .
$$

If we put in this equality $\varphi(s, r)=\left\{\xi_{j} \varphi_{\Lambda}(s, r)\right\}$ where $\xi=\left\{\xi_{j}\right\} \in \mathfrak{S}_{k}$ and $\varphi_{\Lambda}(s, r)$ is the characteristic function of any assigned Borel set $A \subset \Lambda_{k}-A_{k-1}$, then

$$
\int_{\Lambda}\|\xi\|^{2} d \sigma(s) d \rho(r)=\int_{\Lambda}\|M(a ; a s, r) \xi\|^{2} d \sigma(s) d \rho(r) .
$$

This implies that, for any $u_{a} \in \mathrm{U}, M(a ; s, r)$ considered on $\mathfrak{S}_{k}$ is an isometric operator for almost all ${ }^{14)}\langle s, r\rangle \in a\left(\Lambda_{k}-A_{k-1}\right)$. Further, by the definition of

14) Here we mean "for almost all $\langle s, r\rangle$ with respect to the product measure $\sigma \otimes \rho . "$ 
$\imath c_{j k}^{\prime}(a ; s, r)$, the range of $M(a ; s, r)$ is $\mathscr{S}_{k}$ for almost all $\langle s, r\rangle \in\left(\Lambda_{k}-\Lambda_{k-1}\right)$ ( $k$ 른). Since $\Lambda_{1} \subset \Lambda_{2} \subset \ldots$, it follows that for almost all $\langle s, r\rangle \in\left[a\left(\Lambda_{k}-\Lambda_{k-1}\right)\right.$ - $\left.\left(A_{k}-A_{k-1}\right)\right]$ the operator $M(a ; s, r)$ maps $\mathfrak{S}_{k}$ isometrically onto $\mathfrak{F}_{j}$ for some $j \neq k$. Hence every $\left(\Lambda_{k}-\Lambda_{k-1}\right)(k \geq 2)$ must be of the form $S \times \Delta_{k}\left(\Delta_{k} \subset R\right)$ (with the exception of the set of measure zero). On the other hand, $A_{1}$ is of the form $S \times \Delta(\Delta C R)$ from (3.7) and the definition of $A_{1}$. Hence the same is true for every $A_{k}(k=1,2, \ldots)$.

Hereafter we shall say that a matrix $M_{1}(a ; s, r)=\left(u_{j k}^{\mathbb{1}}(a ; s, r)\right)$ is equal to another matrix $M_{2}(a ; s, r)=\left(u_{j k}^{2}(a ; s, r)\right)$ for a. a. (=almost all) $\langle s, r\rangle$ if and only if $u_{j k}^{1}(a ; s, r)=u_{j k}^{2}(a ; s, r)$ for $\sigma \otimes \rho$-almost all $\langle s, r\rangle \notin \Lambda_{k}$ for $j=1$, $2, \ldots, n$; this condition is equivalent to the following one : $M_{1}(a ; s, r)=M_{2}(a$; $s, r)$ as operetors stated in (3.11). By the above obtained result concerning the form of $A_{k}$, if $M_{1}(a ; s, r)=M_{2}(a ; s, r)$ for a. a. $\langle s, r\rangle$ then, for any $b$ $(|b|=1), M_{1}(a ; b s, r)=M_{2}(a ; b s, r)$ for a. a. $\langle s, r\rangle$.

It follows from (3.11) that for any $a, b(|a|=|b|=1)$ and any $\varphi(s, r)$ $=\left\{\varphi_{j}(s, r)\right\}\left(\varphi_{j} \in \Omega_{j}\right)$

$$
M(a ; s, r) \varphi(s, r)=M(b ; s, r) M\left(b^{-1} a ; b^{-1} s, r\right) \varphi(s, r)
$$

as elements of $\mathfrak{M}$. We fix an arbitrary element $u_{a} \in \mathbf{U}$. From (3.12) and by Fubini's theorem, we have

$$
M(a ; s, r)=M(b ; s, r) M\left(b^{-1} a ; b^{-1} s, r\right) \text { for a. a. }\langle b, s, r\rangle .
$$

Since the transformation $\langle b, s, r\rangle \rightarrow\langle s b, s, r\rangle$ is measure-preserving, (3.13) implies that

$$
M(a ; s, r)=M(s b ; s, r) M\left(b^{-1} s^{-1} a ; b^{-1}, r\right) \text { for a. a. }\langle b, s, r\rangle ;
$$

this holds for any fixed $u_{a} \in \mathbf{U}$. Since $\mathbf{U}$ is separable, there exists a countable set $U_{0} \subset \mathbf{U}$ which is dense in $\mathbf{U}$ and contains the identity $e$ of $\mathbf{G}$. Hence we may take an element $b_{0} \in S$ such that

$$
M(a ; s, r)=M\left(s b_{0} ; s, r\right) M\left(b_{0}^{-1}\left(a^{-1} s\right)^{-1} ; b_{0}^{-1}, r\right) \text { for a. a. }\langle s, r\rangle
$$

for all $u_{a} \in \mathbf{U}_{0}$, and that $N_{1}(s, r)=M\left(s b_{0} ; s, r\right)$ and $N_{2}(s, r)=M\left(b_{0}^{-1} s^{-1}, b_{0}^{-1}, r\right)$ are isometric operator for a. a. $\langle s, r\rangle$. Thus we obtain

$$
M(a ; s, r)=N_{1}(s, r) N_{2}\left(a^{-1} s, r\right) \text { for a. a. }\langle s, r\rangle
$$

for all $u_{a} \in \mathrm{U}_{0}$. Putting $u_{a}=e\left(\in \mathbf{U}_{0}\right)$, we get

$$
N_{1}(s, r) N_{2}(s, r)=I \text { for a. a. }\langle s, r\rangle \text {. }
$$

Now put $\psi(s, r)=N_{2}(s, r) \varphi(s, r)$; then $\|\psi(s, r)\|=\|\varphi(s, r)\|$ and $\varphi(s, r)$ $=N_{1}(s, r) \psi(s, r)$ (by (3.15)) for a. a. $\langle s, r\rangle$. And hence, by (3.14) and (3.11), $f \sim \varphi(s, r) \sim \psi(s, r)$ implies 


$$
\left\{\begin{array}{l}
\|f\|_{i}=\int_{\widetilde{x}}\|\varphi(s, r)\|^{2} d \sigma(s) d \rho(r) \\
U_{o} \sim \phi\left(a^{-1} s, r\right) \text { for any } u_{a} \in \mathbf{U}_{0} ; \\
V_{b} \sim(b,\langle s, r\rangle) \psi(s, r) \text { for any } v_{b} \in \mathrm{V} .
\end{array}\right.
$$

By the definition of $\mathfrak{S}_{0}, \psi(s, r)=\left\{\psi_{1}(s, r), \psi_{2}(s, r), \ldots\right\}$, where $\psi_{j}(s, r) \in L^{2}(\tilde{X}$, $\sigma \otimes \rho)$ and $\|\psi(s, r)\|^{2}=\sum_{j=1}^{n}\left|\psi_{j}(s, r)\right|^{2}$ for every $\langle s, r\rangle$. Hence $\mathfrak{M}$ may be realized as a subspace of the direct sum of at most countable number of $L^{2}(\widetilde{X}, \sigma \otimes \rho)$, and $f \sim\left\{\psi_{j}(s, r)\right\}$ implies

$$
\left\{\begin{aligned}
& \text { i) }\|f\|_{\mathfrak{S}}^{2}=\sum_{j=1}^{n} \int_{\widetilde{x}}\left|\psi_{j}(s, r)\right|^{2} d \sigma(s) d \rho(r) \quad(n \leqq \infty) \\
& \text { ii) } U_{a} f \sim\left\{\psi_{j}\left(a^{-1} s, r\right)\right\} \text { for any } u_{a} \in \mathbf{U}_{0} \\
& \text { iii) } V_{b} f \sim\left\{(b,\langle s, r\rangle) \psi_{j}(s, r)\right\} \text { for any } v_{b} \in \mathbf{V} .
\end{aligned}\right.
$$

For any $u_{a} \in \mathbf{U}$, there exists a sequence $\left\{u_{a_{n}}\right\} \subset \mathbf{U}_{0}$ such that $u_{a_{n}} \rightarrow u_{a}$, and $U_{a_{n}} f \sim\left\{\psi_{j}\left(a_{n}^{-1} s, r\right)\right\}$ for any $f \sim\left\{\psi_{j}(s, r)\right\}$. Since the representation $U(g)$ is strongly continuous, we may easily show that $U_{a} f \sim\left\{\psi_{j}\left(a^{-1} s, r\right)\right\}$ for any $f \sim$ $\left\{\psi_{j}(s, r)\right\}$. Namely (3.16) ii) holds for any $u_{n} \in \mathrm{U}$. Hereafter we shall write $\|\cdot\|$ instead of $\|\cdot\|_{5}$.

Let now the cyclic unitary representation $\left\{\check{g}, U(g), f^{\circ}\right\}$ be irreducible. Then either $\mathfrak{M}$ or $\mathfrak{N}$ must be $\{0\}$. If $\mathfrak{M}=\{0\}$, then $\left\{\mathfrak{M}, U_{a}\right\}$ is an irreducible representation of the group $\mathbf{U}$ and $V_{b}=I$ in $\Re$ for all $v_{b} \in V$. Hence the normal elementary $\mathrm{p}$. d. function $\mathscr{D}(g)$ corresponding to the irreducible representation $\{\mathscr{g}, U(g)\}(\mathscr{J}=\mathfrak{R})$ is a character $\chi(a)$ stated in Theorem 1 iii). Conversely such a representation $\{\mathfrak{g}, U(g)\}$ of $\mathbf{G}$ is evidently irreducible. Next suppose that $\mathfrak{N}=\{0\}$; then the unitary space $\mathscr{S}_{0}$ stated above is of one dimension and there exists a point $r_{0} \in R$ such that $\rho\left(\left\{r_{0}\right\}\right)>0$ and $\rho\left(R-\left\{r_{0}\right\}\right)=0$. Hence the irreducible representation $\{\mathscr{S}, U(g)\}$ and the corresponding normal elementary p. d. function are of the form stated in Theorem 1 i). The irreducibility of such representation is proved by means of Lemma 3. Thus, i), iii) and iv) of Theorem 1 is established.

Next we shall prove ii). If the representation $\left\{\mathscr{S}_{1}, U_{1}(g)\right\}$ corresponding to $r_{1}$ is unitary equivalent to $\left\{\mathscr{g}_{2}, U_{2}(g)\right\}$ corresponding to $r_{2}\left(\neq r_{1}\right)$, then $\left(U_{1}(g), f_{1}\right.$, $\left.f_{1}\right)=\left(U_{2}(g) f_{2}, f_{2}\right)$ for certain $f_{1} \in \mathscr{S}_{1}$ and $f_{2} \in \mathscr{S}_{2}$. Hence, if we consider the direct $\operatorname{sum}\left\{\mathscr{S}_{g}, U(g)\right\}=\left\{\mathscr{S}_{1}, U_{1}(g)\right\} \oplus\left\{\mathscr{H}_{2}, U_{2}(g)\right\}$ and put $f=f_{1}+f_{2}$, then $\{U(g) f / g$ $\in \mathbf{G}\}$ does not span $\mathscr{S}$ by Theorem 8 in [3]. But we may prove by Lemma 3 that $\{U(g) f / g \in \mathbf{G}\}$ spans $\mathfrak{S}$. Hence we get Theorem 1 ii).

$\S 4$. Proof of Theorem 2 and supplementary remarks. In this paragraph, we shall make use of the results obtained in $\S 3$. If $\left\{.5, U(g), f^{\circ}\right\}$ is any cyclic unitary representation of $\mathbf{G}$, then the space $\mathscr{F}$ is decomposable to the direct sum of two $U(g)$-invariant subspaces $\mathfrak{R}$ and $\mathfrak{M}$, as stated in $\S 3$; the space $\mathfrak{M}$ is 
realized as the space of $\mathscr{S}_{0}$-valued functions $\psi(s, r)=\left\{\psi_{j}(s, r)\right\}$ on $S \times R$ and the norm $\|f\|$ of the element $f \in M$ and unitary operators $U_{a}$ (for $u_{a} \in \mathbf{U}$ ) and $V_{b}$ (for $v_{b} \in \mathbf{V}$ ) are given by (3.16).

In the case that the cyclic unitary representation $\left\{\delta, U(g), f^{\circ}\right\}$ is not necessarily irreducible, both $\mathfrak{M}$ and $\mathfrak{N}$ may be $\neq\{0\}$. If $\Re \neq\{0\}$, then $\{\mathfrak{R}, U(g)\}$ is a cyclic unitary representation of the group $\mathbf{U}$, and consequently is the direct sum $\underset{k=1}{\oplus}\left\{\mathfrak{M}_{l_{k}}, U_{l_{k}}(g)\right\}(N \leqq \infty)$ as stated in Theorem $\left.2 \mathrm{i}\right)$. If $\mathfrak{M} \neq\{0\}$, then $\{\mathfrak{R}$, $U(g)\}$ is cyclic and is decomposable to the direct sum of $\left.\left\{s_{i}\right\}_{j}, U(g)\right\}, j=1$, $2, \ldots, n(\leqq \infty)$, where $\mathfrak{M}_{j}$ is a subspace of $L^{2}(\widetilde{X}, \sigma \otimes \rho)$ and $U(g)$ is defined by (3.16) for every $j$. If

$$
f^{\circ}=\sum_{j=0}^{n} \psi_{j}^{\circ} \quad \phi_{i}^{\circ} \in \mathfrak{R}, \quad \phi_{j}^{\circ} \in \mathfrak{M}_{j}(j \gtrsim 1),
$$

then $\left\langle\mathfrak{M}_{j}, U(g), \psi_{j}^{\circ}\right\}, j=1,2, \ldots, n$, are cyclic unitary representation of $\mathbf{G}$. Put $J_{j}(r)=\int_{S}\left|\psi_{j}^{\circ}(s, r)\right|^{2} d \sigma(s), \rho_{j}(\Delta)=\int_{\Delta} J_{j}(r) d_{i} \rho(r)$ for $\Delta \subset R$ and

$$
\tilde{\psi}_{j}(s, r)=\left\{\begin{array}{lll}
\psi_{j}(s, r) / J_{j}(r) & \text { if } J_{j}(r) \neq 0 \\
0 & \text { if } J_{j}(r)=0,
\end{array}\right.
$$

and define the unitary operator $U(g)=U_{a} V_{b}$ (for $g=u_{a} v_{b}$ ) by $U_{a} \tilde{\psi}_{j}(s, r)$ $=\tilde{\psi}_{j}\left(a^{-1} s, r\right)$ and $V_{b} \tilde{\psi}_{j}(s, r)=(b,\langle s, r\rangle) \tilde{\psi}_{j}(s, r)$. Then the unitary representation $\left\{L^{2}(\tilde{X}, \sigma \otimes \rho), U(g)\right\}$ (defined by (3.16)) is unitary equivalent to $\left\{L^{2}(\tilde{X}\right.$, $\left.\left.\sigma \otimes \rho_{j}\right), U(g)\right\}$ (defined above) by means of the mapping $\psi_{j}(s, r) \rightarrow \tilde{\psi}_{j}(s, r)$. If we put $f_{j}(s, r)=\widetilde{\psi}_{j}^{o}(s, r)$, then $\left\{U(g) f_{j} / g \in G\right\}$ spans $L^{2}\left(\widetilde{X}, \sigma \otimes \rho_{j}\right)$ by Lemma 3. Hence we may consider that $\mathfrak{M}_{j}=L^{2}\left(\widetilde{X}, \sigma \otimes \rho_{j}\right)$. Clearly the functions $f_{j}(s, r)$, $j=1,2, \ldots$, satisfy the conditions $1^{\circ}$ ) and $2^{\circ}$ ) in Theorem $2 \mathrm{i}$ ). By Theorem 8 in [3], the direct sum $\{\mathfrak{M}, U(g)\}=\bigoplus_{j=1}^{n}\left\{\mathfrak{M}_{j}, U(g)\right\}$ is cyclic if and only if $f_{j}(s, r), j=1,2, \ldots$, satisfy the condition $\left.3^{\circ}\right)$ also. Thus $\left\{\mathscr{S}, U(g), f^{\circ}\right\}$ must be of the form as stated in Theorem 2, and the corresponding p. d. function $\Psi(g)$ is given by (1.5), and consequently (1.6) is evident.

Conversely let us consider the unitary representation $\left\{\mathfrak{g}, U(g), f^{\circ}\right\}$ stated in Theorem $2 \mathrm{i}) . \quad\left\{\mathfrak{M}_{j}, U(g), f_{j}\right\}, j=1,2, \ldots$, are cyclic as stated above. Consequently p. d. functions $\Psi_{j}(g)=\left(U(g) f_{j}, f_{j}\right), j=1,2, \ldots$, are mutually disjoint ${ }^{15)}$ from the assumptions $\left.\left.1^{\circ}\right), 2^{\circ}\right)$ and $\left.3^{\circ}\right)$. Hence the direct sum $\underset{j=1}{\oplus}\left\{\mathfrak{M}_{j}, U(g), f_{j}\right\}$ is cyclic as is earily proved by making use of Theorem 8 in [3]. Similar argument shows that the direct sum $\underset{N}{\stackrel{N}{N}}\left\{\Re_{l_{l_{c}}}, U_{l_{k}}(g)\right\}$ also is cyclic. Since $U_{l_{k}}\left(v_{b}\right)$ $=I$ in $\underset{k=1}{\oplus} \mathfrak{M}_{l_{k}}$ for all $v_{b} \in \mathrm{V}$ and $U\left(v_{b}\right) \equiv V_{b} \neq I$ in $\mathfrak{M}_{j}$ for all $v_{b} \neq e$, we may prove

15) See [3] $\S 12$. 
by making use of Theorem 8 in [3] again that $\left\{\mathscr{g}, U(g), f^{\circ}\right\}$ is a cyclic unitary representation of G. And hence (1.5) follows at once. Thus Theorem 2 is established.

Supplementary remarks. In the proofs of Theorems 1 and 2, we make use of the following fact. The group $\mathbf{G}$ has the property (1.1), where the group $\mathbf{U}$ may be replaced by any group the types of whose unitary representations are well known (for example, a maximally almost periodic Lie group), and either the character group $X$ of the commutative group $\mathbf{V}$ or $\widetilde{X}=X-\left\{\chi_{0}\right\}$ is a topoJogical product space $S \times R$, where $S$ is invariant under the transformation $\chi \rightarrow T_{a} \chi$ defined by $\left(u_{a} v_{b} u_{a}^{-1}, \chi\right)=\left(v_{b}, T_{a} \chi\right)$ and may be considered as a group isomorphic to the group $\mathbf{U}$. The group $\mathbf{G}^{\prime}$ (stated in $\S 0$ ) also satisfies the above conditions.

As for the group of all congruent transformations in the $n$-dimensional euclidean space $E^{n}$ for $n \geqq 3$, the space $S$ is not a group but a factor space $S O(n) / S O(n-1)$ while $\mathbf{U}=S O(n)$. Hence we must consider the space of functions $\psi(u, r)$ on $U \times R$ instead of the space of functions $\psi(s, r)$ on $S \times R$ (in $\S 3$ ). It seems to be difficult to find irreducible invariant subspaces in the space of functions on $\mathbf{U} \times R$, since the similar argument to Lemma 3 is impossible.

\section{LITERATURE}

[1] J. L. Doob: Stochastic processes with an integral-valued parameter, Trans. Amer. Math. Soc. 44 (1938).

[2] I. Gelfand and M. Neumark: Unitary representations of the group of linear transformations of the straight line, C. R. (Doklady) Acad. Sci. URSS. 55 (1947) pp. 567-570.

[3] R. Godement: Les fonctions de type positif et la théorie des groupes, Trans. Amer. Math. Soc. 63, No. 1 (1948) pp. 1-84.

[4] E. Slutsky: Sur les fonctions aléatoires presque périodiques et sur la décomposition des fonctions aléatoires stationaires en composantes, Act. Sci. Ind. 738 (1938).

[5] M. H. Stone : Linear transformations in Hilbert spaces and their applications to analysis, New York (1932).

Mathematical Institute, Nagoya University 